\title{
PERFORMANCE EVALUATION OF A DEVELOPED CASHEW NUT SHELL LIQUID EXPELLER
}

Elijah Oladimeji Aina ${ }^{\mathrm{a}}$, Alex Folami Adisa ${ }^{\mathrm{a}^{*}}$, Tajudeen Mukaila Adeniyi Olayanju $\mathrm{a}^{\mathrm{a}}$ Salami Olasunkanmi ismaila ${ }^{\mathrm{b}}$

a Agricultural and Bioresources Engineering Department, Federal University of Agriculture, Abeokuta, Nigeria

b Mechanical Engineering Department, Federal University of Agriculture, Abeokuta, Nigeria.

'Corresponding author:e-mail: alexadisa@yahoo.co.uk

\begin{tabular}{|c|c|}
\hline ARTICLE INFO & ABSTRACT \\
\hline $\begin{array}{l}\text { Article history: } \\
\text { Received: January } 2018 \\
\text { Received in the revised form: } \\
\text { April } 2018 \\
\text { Accepted: June } 2018 \\
\end{array}$ & \multirow[b]{2}{*}{$\begin{array}{l}\text { The thick vesicant oil liquid contained in the shell of cashew nut, } \\
\text { called cashew nut shell liquid (CNSL), has been known for its innu- } \\
\text { merable applications in chemical industries. Performance evaluation } \\
\text { of a newly developed CNSL expeller was carried out to determine the } \\
\text { effect of moisture content and pressing duration on extraction parame- } \\
\text { ters. The nut shells were grouped as A: } 14.00-16.99 \%, \mathrm{~B}: 17.00 \text { - } \\
19.99 \% \text { and C: } 20.00-22.99 \% \text { (w.b.) moisture content at } 2,4,6,8 \text { and } \\
10 \text { minutes pressing time and combination of } 1,2,3,4 \text { and } 5 \mathrm{~kg} \text { of } \\
\text { cashew nut shells. Analysis of variance (ANOVA) was used for the } \\
\text { stastical analysis. The best mass of liquid extracted, best extraction } \\
\text { efficiency and best percentage oil recovery occured at pressing dura- } \\
\text { tion of } 10 \text { minutes and moisture content of } 14.00-16.99 \% \text { (w.b.), while } \\
\text { the best machine capacity was at pressing duration of } 2 \text { minutes and } \\
\text { moisture content of } 14.00-16.99 \% \text { (w.b.). Results showed that the } \\
\text { effects of the pressing duration and moisture content on mass of liquid } \\
\text { extracted, machine capacity, extraction efficiency and percentage } \\
\text { liquid recovery were significant at } 0.05 \text {. Meanwhile, the effects of the } \\
\text { pressing duration and moisture contents were not significant for the } \\
\text { feed rate. Increase in the pressing duration at decreasingthe moisture } \\
\text { content resulted in the increase in the mass of liquid extracted, extrac- } \\
\text { tion efficiency and percentage liquid recovery while increase in press- } \\
\text { ing duration and moisture content resulted in decrease in machine } \\
\text { capacity of the expeller. }\end{array}$} \\
\hline $\begin{array}{l}\text { Key words: } \\
\text { Cashew nut shell, } \\
\text { liquid expeller, } \\
\text { output capacity, } \\
\text { extraction efficiency }\end{array}$ & \\
\hline
\end{tabular}

\section{Introduction}

Cashew became a popular crop in 1953 when planted on a large scale majorly for the nuts, afforestation and erosion prevention programmes in the escarpment areas of Udi, Mbala, Oghe, Oji, Isuochi and Kingie in the Eastern Nigeria by the defunct Eastern Nigeria Development Corporation (ENDC) (Akinwale et al., 1989). The cultivation at commercial level actually started in 1950s in Western Nigeria by the then Western Nigeria Develop- 
E.O. Aina, A.F. Adisa, T.M.A. Olayanju, S.O. Ismaila

ment Corporation (WNDC) (Togun, 1977). Thereafter, cashew was introduced into the Middle Belt and Northern Nigeria from the Eastern and Western Nigeria and these cashew nuts were majorly of the medium nut-size biotype. This existing cashew biotype (which was introduced from Asian continent) constitutes the landscape of cashew in the Nigerian cashew plantations and attracts low premium in the International market (Rodrigues et al., 1972). Meanwhile, the recent introduction of the Brazilian cashew biotype called 'jumbo nut-size' by the Cocoa Research Institute of Nigeria (CRIN) which is now being grown by cashew farmers, with the cultivation of the crop is rapidly spreading to all agro-ecologies of Nigeria (Topper et al., 2001).

\section{Cashew nut shell}

Cashew nut shell has a bulk density of $481.83 \mathrm{~kg} \cdot \mathrm{m}^{-3}$, thickness of $3.1 \mathrm{~mm}$ with calorific value which is nearly about $5000 \mathrm{kcal} \cdot \mathrm{kg}^{-1}$ and oil content of about 20 to $25 \%$ (Sengar et al., 2012). The conically shaped nut is attached to the lower portion of the cashew apple and it hangs at the bottom of the apple. In its natural state, the shell of the cashew nut is leathery and not brittle and it contains the thick vesicant oil called 'cashew nut shell liquid' (CNSL), within a sponge -like interior. The kernel is surrounded by a thin testa skin which keeps it separated from the inside of the shell.The surface area, pore volume and average pore diameter of the cashew nut shell as determined by Brunauer et al., (1999) using a surface area and pore size analyzer were $395 \mathrm{~m}^{2} \cdot \mathrm{g}^{-1}, 0.4732 \mathrm{~cm}^{3} \cdot \mathrm{g}^{-1}, 5.89 \mathrm{~mm}$, and $0.415 \mathrm{~g} \cdot \mathrm{cm}^{-1}$ respectively.

\section{Cashew nut shell liquid (CNSL)}

The shell is formed of three concentric layers, the epicarp, the mesocarp, and the endocarp. The mesocarp is spongy and comprised of a mass of sclerenchymatic tissues and ducts containing about $35 \%$ of sticky, resinous liquid called cashew nut shell liquid (CNSL). The CNSL is highly caustic-brown in colour and inedible. Its role is probably to afford the fruit some natural protection against insects. CNSL consists of two highly reactive phenolic compounds which are anarcardic acid $(90 \%)$ and cardol $(10 \%)$. Cardol is used in colouring fish nets, painting furniture, books and other stationary to prevent the attack of insects, especially the silver fish. It is also used as external application for leprosy and ringworm (Mandal, 2012). CNSL has innumerable applications, such as friction linings, paints, laminating resins, rubber compounding resins, cashew cements, polyurethane based polymers, surfactants, epoxy resins, foundry chemicals, and intermediates for chemical industry. It offers much scope and varied opportunities for the development of other tailor-made polymers (Akinhanmi et al., 2008). CNSL undergoes all the conventional reactions of phenols, CNSL aldehyde condensation products and CNSL based phenols. Resins are used in applications such as surface coatings and adhesives. Various polyamines synthesised from CNSL are used as curing agents for epoxy resins. CNSL and its derivatives have been used as antioxidants, plasticizers and processing aids for rubber compounds, modifiers for plastic materials and used to provide oxidative resistance sulphur-cured natural rubber products. It is also added to rubber gum stock or nitrile rubber to improve its process ability, mechanical properties and resistance to crack and cut properties of the vulcanisates (Akinhanmi et al., 2008). 
Performance evaluation...

\section{Cashew nut processing}

Processing of cashew nuts can be defined as the recovery of the kernel (edible matter portion) from the raw nuts by manual or mechanical means. It consists of moisture conditioning, shelling, drying, peeling, grading and packing (Mandal, 2012). The nut consists of a coriaceous epicarp, spongy mesocarp containing a viscous resinous liquid and stony endocarp, that is, white kernel covered with a thin testa membrane. The processing of the nuts may be done by manual method or through the factory processing.

\section{(a) Manual method}

The manual way consists in burning of raw nuts for a short time on an open fire or roasting in a pan, just enough to burn the outer shell and drive off the volatile shell liquid (CNSL) which is usually ignited. After cooling, the shells are cracked with a small wooden rod and the kernels are extracted. In this process, scorching of the part of the kernels (particularly in tips) is almost inevitable and hence such kernels are suitable only for domestic consumption but not fit for export market since the product is of poor quality, often contaminated by the vesicant shell liquid (Manual, 2012). The open pan roasting is still common in Tamil Nadu, West Bengal and Kerala but gradually disappearing (Mandal, 2012).

\section{(b) Industrial processing}

Avoiding the contamination of kernels by the toxic cashew nut shell liquid (CNSL) and minimising kernel breakage are the two major considerations in the decortication of the cashew nuts. Hence, the roasting is to be done to make the shell brittle, and then the shell must be broken in such a way that the whole kernel can be removed to fetch the export market (Mandal, 2012). This operation is tricky and requires patience, skill and practice. After removing the small foreign particles through sieving, the cleaned nuts are calibrated into big, medium and small size groups because the nuts of different sizes require timing for humidification and roasting. Criteria for the size are width and thickness, not length. Then, conditioning takes place (this is the fixation of the moisture level which renders the shells soft and facilitates the rupture of the oil-bearing cells). Conditioning should not increase the moisture content and water temperature too much, otherwise, tanning of the peel may penetrate into the kernel, affecting its colour and value as well it may receive low prices in the market. Hence, the nuts must be conditioned at optimum bursting of CNSLcontaining cells during the roasting process (Mandal, 2012). At the optimum moisture content, the kernels become rubbery and thus reduce the chance of breaking during roasting. For conditioning, the nuts brought from a store are kept in heaps and thoroughly soaked in water by regularly spraying for two days, covered with moist gunny bags and providing water to drain from the floor immediately. Then the nuts are considered to be moist enough for roasting (15-20\% desired moisture level) stated by Mandal (2012). The conditioned nuts are then roasted by different methods which include open pan, drum, oil bath and mild after which shelling (the process of removing the shells from the cashew nuts) takes place.

Performance evaluation of newly developed agricultural machinery is very important to determine physical performance specifications for its effective utilization (Adisa, 2011). A mechanical cashew nut shell liquid expeller was newly developed and thorough performance evaluation was carried out to determine its critical operating parameters which was 
the main aim of this study. It is a project for turning cashew nut shell waste to wealth, that is, cashew nut shell which used to be discarded as waste and burnt after extracting juice from the fruit and cashew nuts removed for domestic and industrial usage can now produce additional by-product of CNSL.

\section{Materials and Methods}

\section{Operation of developed expeller}

The developed expeller makes use of a tapering auger as shown in Figures 1, 2 and 3 that is, the entrance opening is bigger and opening kept reducing towards the outlet thereby compressing the nut shell to release the liquid content and pushes the cashew nut shell that is fed into it through the hopper along a barrel - shaped outer casing (expression chamber) with a gradually decreasing cross-sectional area towards the taper end.

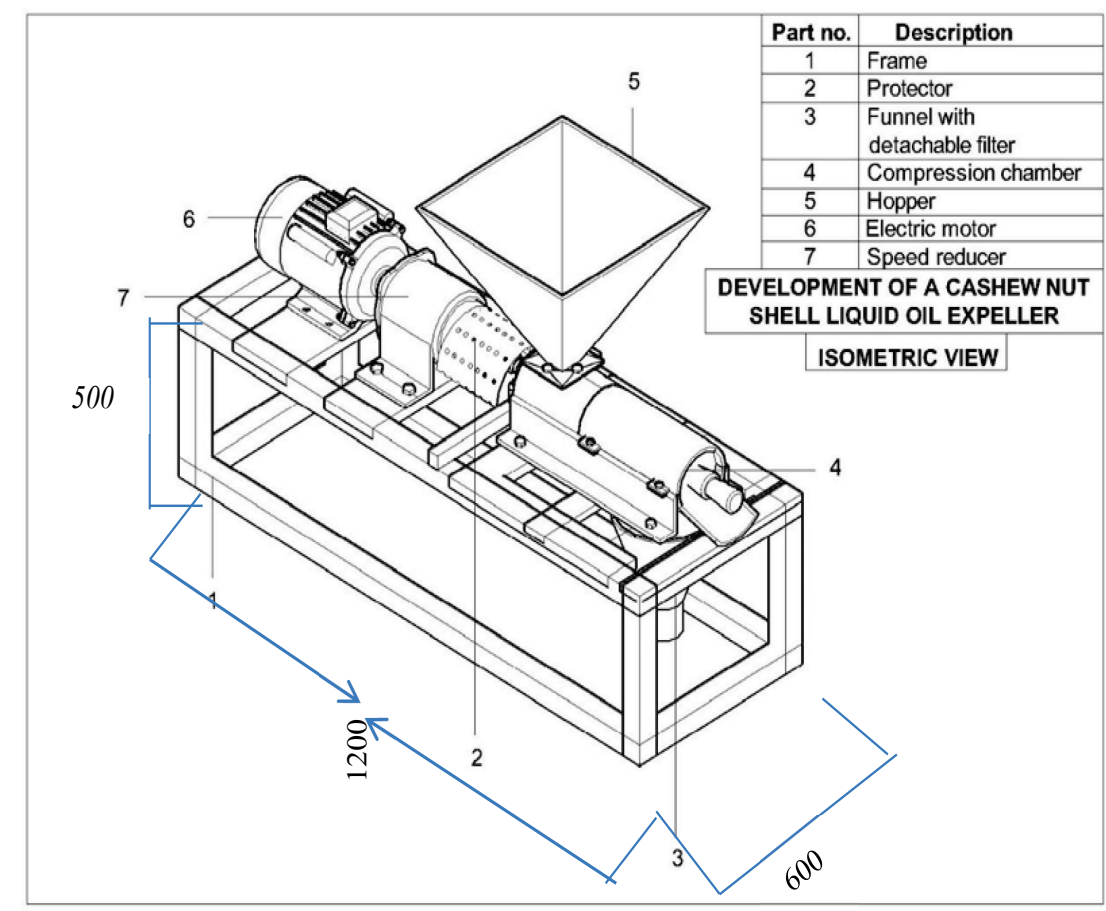

Figure 1. Isometric view of the cashew nut shell liquid expeller 
Performance evaluation...

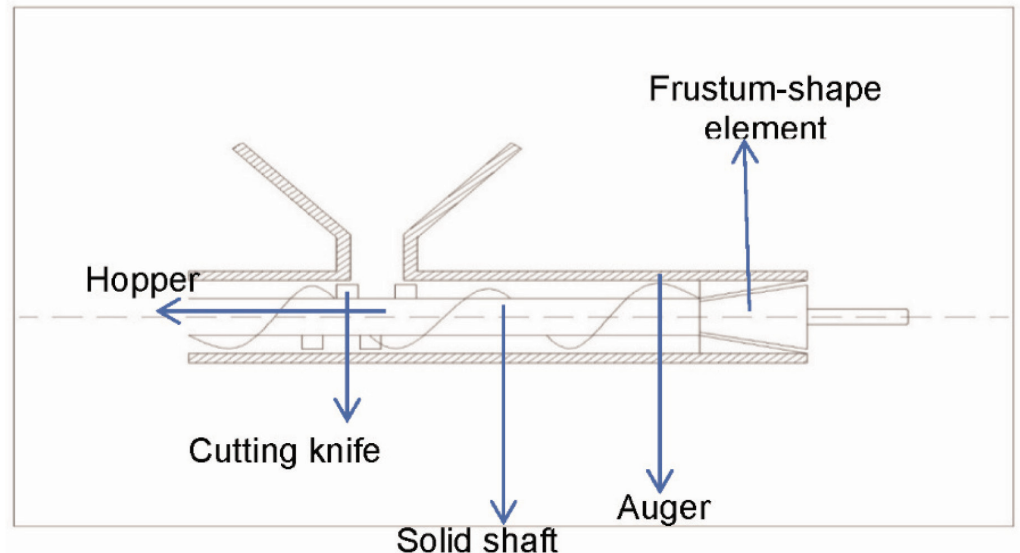

Figure 2. Cross-sectional view of the cashew nut shell liquid expeller's compression chamber

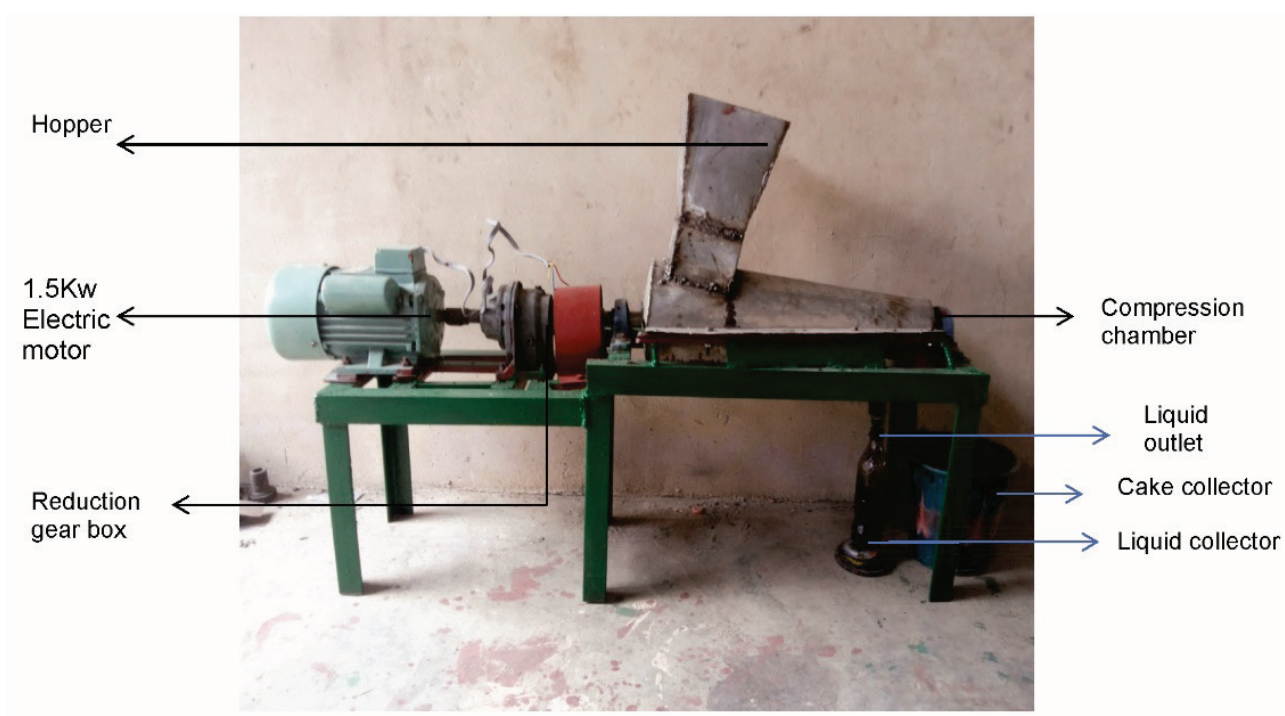

Figure 3. Fabricated cashew nut shell liquid expeller

Beneath the hopper and on the taper auger is a set of knives ( $2 \mathrm{~mm}$ thick) that helped reduce the surface area (size) of the cashew nut shell whose liquid is to be expressed, this is to allow for better expression of liquid from the shell. At the end portion of the cylinder is an abrupt frustum-shaped element which greatly increased the pressure on the product to $0.25 \mathrm{MPa}$ as it is conveyed to the fulcrum shaped shaft end where the major squeezing occurred thereby rupturing the liquid cells in the cashew nut shell and causing liquid to 
E.O. Aina, A.F. Adisa, T.M.A. Olayanju, S.O. Ismaila

flow through the perforated walls in the casing (incorporated with a wire gauze to aid primary level offiltration) which was then collected in a trough (incorporated with secondary level of filtration material) underneath the perforated walls. The cake (residue) from the compressed cashew nut shell from which liquid has been extracted and exits the unit through the cake outlet and this allows for greater liquid expression.

\section{Experimental procedure}

The cashew nut shells were cleaned, sampled and prepared for tests. The shells were handled separately but were given the same pre-treatment of moisture conditioning to 14$16.99 \%$ (group A), $17-19.99 \%$ (group B) and $20-22.99 \%$ (group C) all on the wet basis for time interval of 2, 4, 6, 8 and 10 minutes. Conditioning of samples was done by drying the measured sample at $103^{\circ} \mathrm{C}$ for 5 hours (ASAE, 1998) until there was a weight constant and a measured quantity of water was added to the samples to give it the required moisture content using equation 1 as given by Akinoso (2006). Each sample was sealed in a separate polythylene film after the addition of distil water. The samples were kept at $5^{\circ} \mathrm{C}$ in a refrigerator for a day to enable the moisture distribute uniformly throughout the samples.

$$
\mathrm{Q}=\frac{\mathrm{A}(\mathrm{kg})(\mathrm{b}-\mathrm{a})(\%)}{(100-\mathrm{b})(\%)}
$$

where:

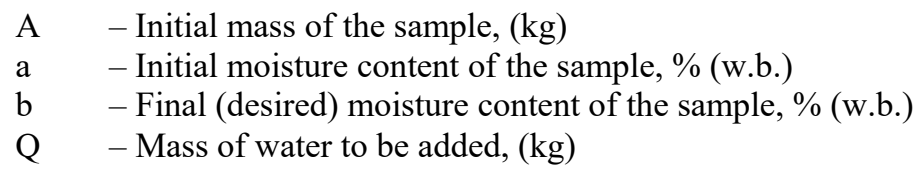

For each treatment combination, fifteen samples each of 1,2, 3, 4 and $5 \mathrm{~kg}$ of cashew nut shell were used to carry out the tests and this was done in two replicates. Stop watch was used to monitor the pressing duration and at the end of the extraction, the quantity of liquid obtained was recorded.

The performance indices at the various moisture content were evaluated. These were oil recovery efficiency, oil extraction efficiency, machine capacity of the expeller that were carried out using analysis of variance (ANOVA) in order to determine the extent to which the moisture content and pressing duration affected affected the performance indices. Statistical package for social sciences, SPSS version 20.0 by IBM SPSS statistics, Armonk, New York, USA was suited for the two factor treatments.

\section{Performance evaluation}

Mass of liquid extracted (ML) at moisture content A, B, C, ML ( $\mathrm{kg}$ ) was calculated by equation 2, Ojomo et al. (2012)

$$
\mathbf{M L}=\mathrm{M}_{\mathrm{CL}}-\mathrm{M}_{\mathrm{C}}
$$


Performance evaluation...

Feed rate (FR) at moisture content $\mathrm{A}, \mathrm{B}, \mathrm{C}, \mathrm{FR}\left(\mathrm{kgmin}^{-1}\right)$ was calculated by equation 3, Ojomo et al. (2012)

$$
\mathbf{F R}=\frac{M c n s}{T t}
$$

Machine capacity (MC) at moisture content A, B, C, MC $\left(\mathrm{kgmin}^{-1}\right)$ was calculated by equation 4, Ojomo et al. (2012)

$$
\mathrm{MC}=\frac{M l e}{T t}
$$

Extraction efficiency (EE) at moisture content A, B, C, EE (\%) was calculated by equation 5, Ojomo et al. (2012)

$$
\mathbf{E E}=\frac{\operatorname{Mlex} 100}{\text { Mcns }}
$$

Percentage liquid recovery (PR) at moisture content levels A, B, C, PR (\%) was calculated by equation 6 , Ojomo et al. (2012)

$$
\mathbf{P R}=\frac{E E x 25 \%}{100}
$$

where:

A $-14-16.99 \%$ (w.b.)

b $\quad-17-19.99 \%$ (w.b.)

c $\quad-20-22.99 \%$ (w.b.)

$\mathrm{m}_{\mathrm{CL}}-$ Mass of container and liquid, $(\mathrm{kg})$

$\mathrm{M}_{\mathrm{C}}$ - Mass of container, $(\mathrm{kg})$

$\mathrm{M}_{\mathrm{cns}}-$ Mass of cashew nut shell, $(\mathrm{kg})$

$\mathrm{t}_{\mathrm{t}} \quad$ - Time taken, (min)

$\mathrm{m}_{\mathrm{le}}-$ Mass of liquid expelled, $(\mathrm{kg})$

The oil content in $\mathrm{kg}$ of cashew nut shell is $25 \%$ (Mazzetto et al., 2009)

\section{Results and Discussion}

The cashew nut shell liquid expression was done by varying the mass of the nut shell and time of operation. The mass of liquid extracted, feed rate, extraction efficiency, machine capacity and percentage liquid recovery were recorded.

Analysis of variance (ANOVA) was used to determine the extent to which moisture content and pressing duration affected the performance indices. The F-ratio obtained from the ANOVA of the performance tests results are presented in Table 1. 
E.O. Aina, A.F. Adisa, T.M.A. Olayanju, S.O. Ismaila

Table 1 .

F-ratio for the results of performance tests with cashew nut shell

\begin{tabular}{|c|c|c|c|c|c|c|c|c|c|c|c|c|}
\hline \multirow[t]{2}{*}{$\begin{array}{l}\text { Source } \\
\text { of varia- } \\
\text { tion }\end{array}$} & \multicolumn{3}{|c|}{$\begin{array}{l}\text { Mass of liquid } \\
\text { extracted (ML) }\end{array}$} & \multirow{2}{*}{ 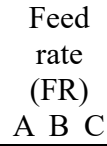 } & \multicolumn{3}{|c|}{$\begin{array}{c}\text { Machine capacity } \\
\text { (MC) }\end{array}$} & \multicolumn{3}{|c|}{$\begin{array}{l}\text { Extraction efficiency } \\
\text { (EE) }\end{array}$} & \multicolumn{2}{|c|}{$\begin{array}{l}\text { Percentage of } \\
\text { liquid recovery } \\
\text { (PR) }\end{array}$} \\
\hline & A & B & $\mathrm{C}$ & & A & B & $\mathrm{C}$ & A & B & $\mathrm{C}$ & A & $\mathrm{C}$ \\
\hline
\end{tabular}

Time

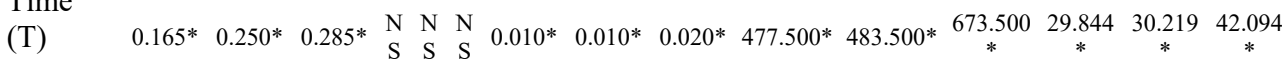

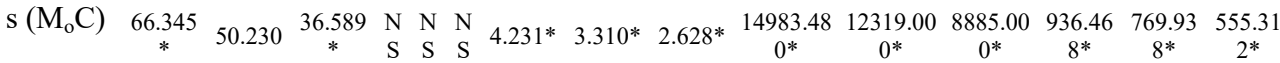

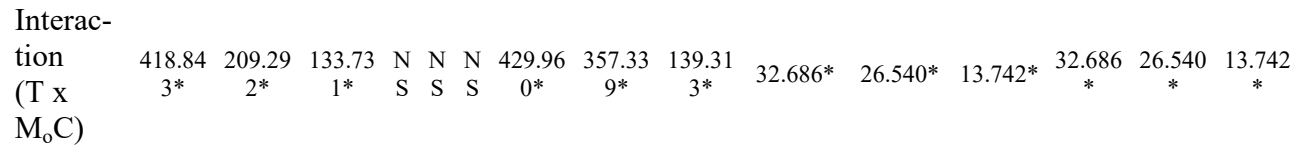

* Significant at 5\% level; FR, Feed rate; NS, Not significant

Table 2.

ANOVA of the performance test results

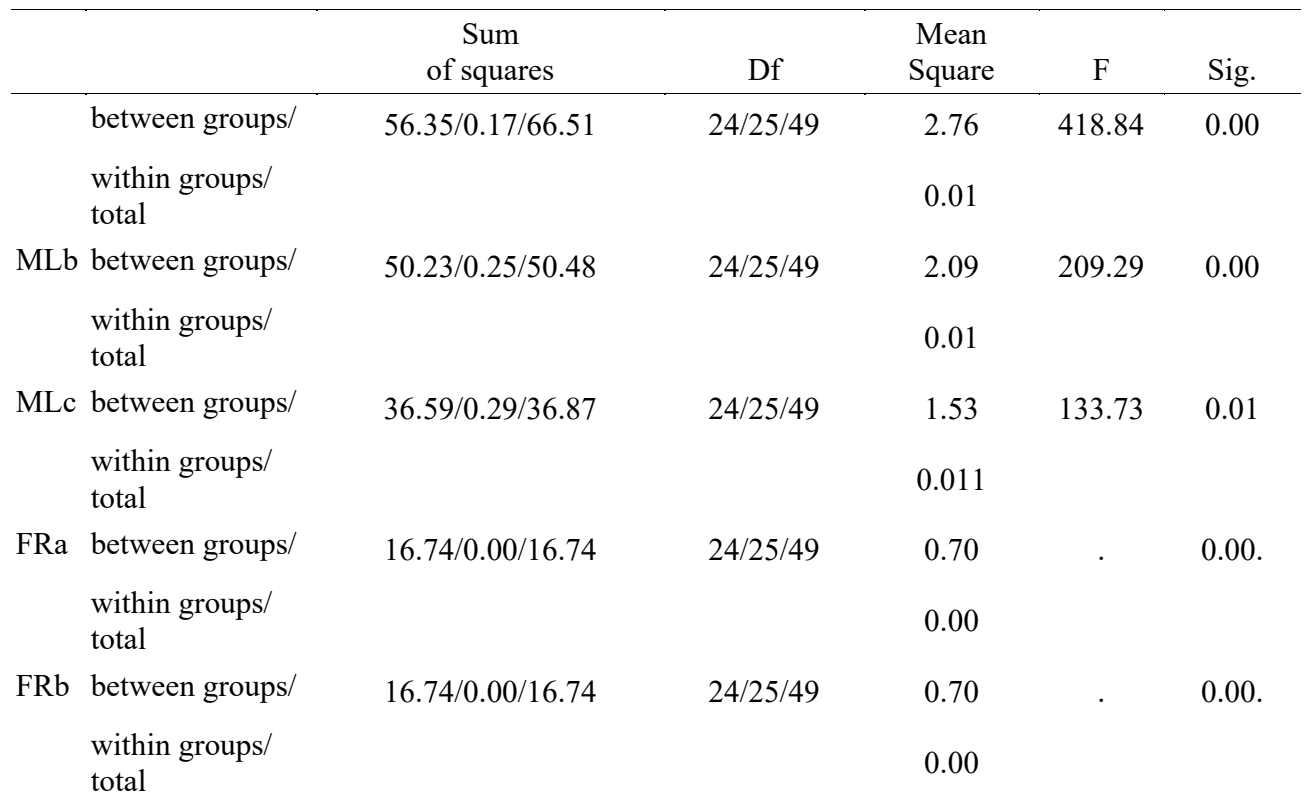


Performance evaluation...

\begin{tabular}{|c|c|c|c|c|c|c|}
\hline & & $\begin{array}{c}\text { Sum } \\
\text { of squares }\end{array}$ & Df & $\begin{array}{c}\text { Mean } \\
\text { Square }\end{array}$ & $\mathrm{F}$ & Sig. \\
\hline FRc & $\begin{array}{l}\text { between groups/ } \\
\text { within groups/ } \\
\text { total }\end{array}$ & $16.74 / 0.00 / 16.74$ & $24 / 25 / 49$ & $\begin{array}{l}0.70 \\
0.00\end{array}$ & . & 0.00 . \\
\hline $\mathrm{MCa}$ & $\begin{array}{l}\text { between groups/ } \\
\text { within groups/ } \\
\text { total }\end{array}$ & $4.23 / 0.01 / 4.24$ & $24 / 25 / 49$ & $\begin{array}{l}0.18 \\
0.00\end{array}$ & 429.96 & 0.00 \\
\hline $\mathrm{MCb}$ & $\begin{array}{l}\text { between groups/ } \\
\text { within groups/ } \\
\text { total }\end{array}$ & $3.31 / 0.01 / 3.32$ & $24 / 25 / 49$ & $\begin{array}{l}0.14 \\
0.00\end{array}$ & 357.34 & 0.00 \\
\hline $\mathrm{MCc}$ & $\begin{array}{l}\text { between groups/ } \\
\text { within groups/ } \\
\text { total }\end{array}$ & $2.63 / 0.02 / 2.65$ & $24 / 25 / 49$ & $\begin{array}{l}0.11 \\
0.00\end{array}$ & 139.31 & 0.00 \\
\hline $\mathrm{EEa}$ & $\begin{array}{l}\text { between groups/ } \\
\text { within groups/ } \\
\text { total }\end{array}$ & $14983.48 / 477.50 / 15460.98$ & $24 / 25 / 49$ & $\begin{array}{l}624.31 \\
19.10\end{array}$ & 32.69 & 0.00 \\
\hline $\mathrm{EEb}$ & $\begin{array}{l}\text { between groups/ } \\
\text { within groups/ } \\
\text { total }\end{array}$ & $12319.00 / 483.50 / 12802.50$ & $24 / 25 / 49$ & $\begin{array}{r}513.29 \\
19.34\end{array}$ & 26.54 & 0.00 \\
\hline $\mathrm{EEc}$ & $\begin{array}{l}\text { between groups/ } \\
\text { within groups/ } \\
\text { total }\end{array}$ & $8885.00 / 673.50 / 958.50$ & $24 / 25 / 49$ & $\begin{array}{l}370.21 \\
26.940\end{array}$ & 13.74 & 0.00 \\
\hline PRa & $\begin{array}{l}\text { between groups/ } \\
\text { within groups/ } \\
\text { total }\end{array}$ & $936.47 / 29.84 / 966.31$ & $24 / 25 / 49$ & $\begin{array}{l}39.02 \\
1.19\end{array}$ & 32.69 & 0.00 \\
\hline $\mathrm{PRb}$ & $\begin{array}{l}\text { between groups/ } \\
\text { within groups/ } \\
\text { total }\end{array}$ & $769.94 / 30.22 / 800.16$ & $24 / 25 / 49$ & $\begin{array}{l}32.08 \\
1.21\end{array}$ & 26.54 & 0.00 \\
\hline PRc & $\begin{array}{l}\text { between groups/ } \\
\text { within groups/ } \\
\text { total }\end{array}$ & $555.31 / 42.09 / 597.41$ & $24 / 25 / 49$ & $\begin{array}{l}23.14 \\
1.68\end{array}$ & 13.74 & 0.00 \\
\hline
\end{tabular}


E.O. Aina, A.F. Adisa, T.M.A. Olayanju, S.O. Ismaila

Table 3.

Duncan multiple range test for mass of liquid extracted, feed rate, machine capacity, extraction efficiency and percentage of oil recovery at different moisture content

\begin{tabular}{|c|c|c|c|c|c|c|c|c|c|c|c|c|c|c|}
\hline Mass & MLa & MLb & MLc & Fra & FRb FRc & $\mathrm{MCa}$ & $\mathrm{MCb}$ & MCc & $\mathrm{EEa}$ & $\mathrm{EEb}$ & $\mathrm{EEc}$ & $\mathrm{PRa}$ & PRb & PRc \\
\hline $\operatorname{kg} 12$ & $0.25^{\mathrm{a}}$ & $0.15^{\mathrm{a}}$ & $0.10^{\mathrm{a}}$ & 0.50 & 0.500 .50 & $0.13^{\mathrm{b}}$ & $0.08^{\mathrm{a}}$ & $0.05^{\mathrm{a}}$ & $25.00^{\mathrm{b}}$ & $15.00^{\mathrm{a}}$ & $10.00^{\mathrm{a}}$ & $6.25^{\mathrm{a}}$ & $3.75^{\mathrm{a}}$ & $2.50^{\mathrm{a}}$ \\
\hline $\operatorname{gg} 14$ & $0.30^{\mathrm{ab}}$ & $0.25^{\mathrm{ab}}$ & $0.15^{\mathrm{ab}}$ & 0.25 & 0.250 .25 & $0.08^{\mathrm{a}}$ & $0.07^{\mathrm{abc}}$ & $0.04^{\mathrm{a}}$ & $30.00^{\mathrm{b}}$ & $25.00^{\mathrm{b}}$ & $15.00^{\mathrm{ab}}$ & $7.50^{\mathrm{b}}$ & $6.25^{\mathrm{b}}$ & $3.75^{\mathrm{ab}}$ \\
\hline $\mathrm{kg} 16$ & $0.45^{\text {bcd }}$ & $0.35^{\text {abc }}$ & $0.25^{\mathrm{abc}}$ & 0.17 & $\begin{array}{lll}0.17 & 0.17\end{array}$ & $0.08^{\mathrm{a}}$ & $0.06^{\mathrm{abc}}$ & $0.04^{\mathrm{a}}$ & $45.00^{\mathrm{cd}}$ & $35.00^{\mathrm{c}}$ & $25.00^{\mathrm{bcd}}$ & $11.25^{\mathrm{cd}}$ & $8.75^{\mathrm{c}}$ & $6.25^{\mathrm{bcd}}$ \\
\hline g18 & $0.50^{\mathrm{cd}}$ & $0.45^{\mathrm{bc}}$ & $0.35^{\mathrm{bcd}}$ & 0.13 & 0.130 .13 & $0.07^{\mathrm{a}}$ & $0.06^{\mathrm{ab}}$ & $0.05^{\mathrm{a}}$ & $50.00^{\text {csde }}$ & $45.00 \mathrm{cdef}$ & $35.00^{\mathrm{det}}$ & $12.50^{\text {cde }}$ & $11.25^{\mathrm{cdef}}$ & $8.75^{\operatorname{def}}$ \\
\hline $\mathrm{kg} 110$ & $0.55^{\mathrm{d}}$ & $0.45^{\mathrm{bc}}$ & $0.35^{\mathrm{bcd}}$ & 0.10 & 0.100 .10 & $0.06^{\mathrm{a}}$ & $0.05^{\mathrm{a}}$ & $0.04^{\mathrm{a}}$ & $55.00^{\operatorname{detg}}$ & $45.00^{\text {cdet }}$ & $35.00^{\mathrm{def}}$ & $13.75^{\text {detg }}$ & $11.25^{\text {cdef }}$ & $8.75^{\mathrm{def}}$ \\
\hline kg22 & $0.35^{\mathrm{abc}}$ & $0.25^{\mathrm{ab}}$ & $0.40^{\text {cde }}$ & 1.00 & 1.001 .00 & $0.18^{\mathrm{c}}$ & $0.13^{\mathrm{d}}$ & $0.20^{\mathrm{bcd}}$ & $17.50^{\mathrm{a}}$ & $12.50^{\mathrm{a}}$ & $20.00^{\mathrm{abc}}$ & $4.38^{\mathrm{a}}$ & $3.13^{\mathrm{a}}$ & $5.00^{\mathrm{c}}$ \\
\hline $\mathrm{g} 24$ & $0.55^{\mathrm{d}}$ & $0.50^{\mathrm{c}}$ & $0.60^{\mathrm{e}}$ & 0.50 & $\begin{array}{lll}0.50 & 0.50\end{array}$ & $0.14^{\mathrm{b}}$ & $0.13^{\mathrm{d}}$ & $0.15^{\mathrm{b}}$ & $27.50^{\mathrm{b}}$ & $25.00^{\mathrm{b}}$ & $30.00^{\text {cde }}$ & $6.88^{\mathrm{b}}$ & $6.25^{\mathrm{b}}$ & $7.50^{\mathrm{cd}}$ \\
\hline $\mathrm{g} 26$ & $0.85^{\mathrm{e}}$ & $0.50^{\mathrm{c}}$ & $0.55^{\mathrm{de}}$ & 0.33 & $\begin{array}{lll}0.33 & 0.33\end{array}$ & $0.14^{\mathrm{b}}$ & $0.09^{\mathrm{abcd}}$ & $0.09^{\mathrm{a}}$ & $42.50^{\mathrm{c}}$ & $25.00 \mathrm{~b}$ & $27.50^{\mathrm{cd}}$ & $10.63^{\mathrm{c}}$ & $6.25^{\mathrm{b}}$ & $6.88^{\mathrm{cd}}$ \\
\hline $\lg 28$ & $1.15^{\mathrm{f}}$ & $0.75^{\mathrm{d}}$ & $0.60^{\text {ef }}$ & 0.25 & 0.250 .25 & $0.15^{\mathrm{b}}$ & $0.10^{\mathrm{bcd}}$ & $0.08^{\mathrm{a}}$ & $57.50^{\text {etg }}$ & $37.50^{\mathrm{cd}}$ & $30.00^{\text {cde }}$ & $14.38^{\text {etg }}$ & $9.38^{\mathrm{cd}}$ & $7.50^{\mathrm{cd}}$ \\
\hline g210 & $1.35^{\mathrm{g}}$ & $1.05^{\mathrm{e}}$ & $0.80^{\mathrm{f}}$ & 0.20 & $\begin{array}{lll}0.20 & 0.20\end{array}$ & $0.14^{\mathrm{b}}$ & $0.11^{\mathrm{d}}$ & $0.08^{\mathrm{a}}$ & $67.50^{\mathrm{hi}}$ & $52.50^{\text {figh }}$ & $40.00^{\text {etg }}$ & $16.88^{\mathrm{hi}}$ & $13.13^{\text {tgh }}$ & $10.00^{\text {efg }}$ \\
\hline $\mathrm{g} 32$ & $1.35^{\mathrm{g}}$ & $1.20^{\mathrm{ef}}$ & $1.10^{\mathrm{g}}$ & 1.50 & 1.50 & $0.68^{\mathrm{k}}$ & $0.60^{\mathrm{k}}$ & $0.55^{\mathrm{i}}$ & $45.00^{\mathrm{cd}}$ & $40.00^{\text {cde }}$ & $37.00^{\operatorname{detg}}$ & $11.25^{\mathrm{cd}}$ & $10.00^{\text {cdef }}$ & $9.25^{\mathrm{fg}}$ \\
\hline $\mathrm{kg} 34$ & $1.55^{\mathrm{h}}$ & $1.35^{\mathrm{tg}}$ & $1.20^{\mathrm{gh}}$ & 0.75 & 0.75 & $0.39^{\text {gh }}$ & $0.34^{\mathrm{h}}$ & $0.30^{\text {ef }}$ & $51.50^{\text {cdef }}$ & $45.00^{\text {cdef }}$ & $40.00^{\text {etg }}$ & $12.88^{\text {cdef }}$ & $11.25^{\mathrm{cdef}}$ & $10.00^{\text {etg }}$ \\
\hline $\mathrm{kg} 36$ & $1.85^{\mathrm{i}}$ & $1.50^{\mathrm{g}}$ & $1.35^{\mathrm{h}}$ & 0.50 & 0.500 .50 & $0.31^{\text {ef }}$ & $0.25^{\mathrm{fg}}$ & $0.23^{\mathrm{cd}}$ & $61.50^{\text {fgh }}$ & $50.00^{\text {efg }}$ & $45.00^{\text {fghi }}$ & $15.38^{\mathrm{fg}}$ & $12.50^{\text {efg }}$ & $11.25^{1}$ \\
\hline g38 & $2.15^{\mathrm{i}}$ & $1.75^{\mathrm{h}}$ & $1.60^{\mathrm{i}}$ & 0.40 & $0.40 \quad 0.40$ & $.2700^{\mathrm{de}}$ & $0.22^{\mathrm{ef}}$ & $0.20^{\text {bcd }}$ & $71.50^{\text {hij }}$ & $58.50^{\text {ghi }}$ & $53.50^{\text {hijk }}$ & $17.88^{\text {hij }}$ & $14.63^{\text {ghi }}$ & $13.38^{\mathrm{hijk}}$ \\
\hline g310 & $2.35^{\mathrm{kl}}$ & $2.05^{\mathrm{i}}$ & $1.80^{\mathrm{ij}}$ & 0.30 & 0.300 .30 & $0.24^{\mathrm{d}}$ & $0.21^{\mathrm{e}}$ & $0.18^{\mathrm{bc}}$ & $78.50^{\mathrm{k}}$ & $68.50^{j}$ & $60.00^{\mathrm{jk}}$ & $19.63^{\mathrm{jk}}$ & $17.13^{\mathrm{jk}}$ & $15.00^{\mathrm{jk}}$ \\
\hline $\mathrm{g} 42$ & $1.95^{\mathrm{i}}$ & $1.75^{\mathrm{h}}$ & $1.60^{\mathrm{i}}$ & 2.00 & 2.002 .00 & $0.98^{1}$ & $0.88^{1}$ & $.80^{\mathrm{j}}$ & $49.00^{\text {cde }}$ & $44.00^{\text {cdef }}$ & $40.50^{\text {efg }}$ & $12.25^{\text {cde }}$ & $11.00^{\text {cdef }}$ & $10.13^{\text {efg }}$ \\
\hline $\operatorname{gg} 44$ & $2.25^{\mathrm{jm}}$ & $1.95^{\mathrm{hi}}$ & $1.75^{\mathrm{ij}}$ & 1.00 & 1.001 .00 & $0.57^{\mathrm{j}}$ & $0.49^{\mathrm{j}}$ & $0.44^{\mathrm{h}}$ & $54.00^{\operatorname{defg}}$ & $49.00^{\text {efg }}$ & $44.00^{\text {fghi }}$ & $13.50^{\text {defg }}$ & $12.25^{\mathrm{cdef}}$ & $11.00^{\text {fghi }}$ \\
\hline kg46 & $2.55^{\mathrm{jkm}}$ & $2.15^{\mathrm{ijk}}$ & $1.90^{\mathrm{jk}}$ & 0.67 & 0.670 .67 & $0.43^{\mathrm{h}}$ & $0.36^{\mathrm{h}}$ & $0.32^{\mathrm{ef}}$ & $64.00^{\mathrm{gh}}$ & $54.00^{\text {fgh }}$ & $48.00^{\text {ghij }}$ & $16.00^{\text {ghi }}$ & $13.50^{\mathrm{fgh}}$ & $12.00^{\text {ghij }}$ \\
\hline $\mathrm{kg} 48$ & $2.80^{\mathrm{n}}$ & $2.30^{\mathrm{jkl}}$ & $2.10^{\mathrm{kl}}$ & 0.50 & 0.50 & $0.35^{\mathrm{fg}}$ & $0.29^{\mathrm{g}}$ & $0.26^{\mathrm{de}}$ & $70.50^{\text {hij }}$ & $57.50^{\text {ghi }}$ & $53.00^{\text {hijk }}$ & $17.63^{\mathrm{hij}}$ & $14.38^{\text {gh }}$ & $13.25^{\mathrm{l}}$ \\
\hline kg410 & $05^{\text {op }}$ & $2.45^{1}$ & $2.20^{\mathrm{lm}}$ & 0.40 & 0.40 & $0.31^{\mathrm{e}}$ & $0.25^{\mathrm{ef}}$ & $0.22^{\text {cd }}$ & & & $55.00^{\mathrm{ijk}}$ & & & \\
\hline $\mathrm{g} 52$ & $2.45^{\mathrm{lm}}$ & $2.10^{\mathrm{ij}}$ & $1.85^{\mathrm{j}}$ & 2.50 & $2.50 \quad 2.50$ & $1.23^{\mathrm{m}}$ & $1.05^{\mathrm{m}}$ & $0.93^{\mathrm{k}}$ & $49.00^{\text {cde }}$ & $42.00^{\text {cde }}$ & $37.00^{\text {detg }}$ & $12.25^{\text {cde }}$ & $10.50^{\text {cde }}$ & $9.25^{\text {defg }}$ \\
\hline $\mathrm{g} 54$ & $2.80^{\mathrm{n}}$ & $2.35^{\mathrm{kl}}$ & $2.10^{\mathrm{kl}}$ & 1.25 & $1.25 \quad 1.25$ & $0.71^{\mathrm{k}}$ & $0.59^{\mathrm{k}}$ & $0.53^{i}$ & $56.00^{\text {etg }}$ & $47.00^{\mathrm{def}}$ & $42.00^{\text {etgh }}$ & $14.00^{\text {etg }}$ & $11.75^{\mathrm{def}}$ & $10.50^{\mathrm{et}}$ \\
\hline g56 & $3.55^{\mathrm{kl}}$ & $2.90^{\mathrm{m}}$ & $2.35^{\mathrm{m}}$ & 0.83 & $\begin{array}{lll}0.83 & 0.83\end{array}$ & $0.59^{\mathrm{j}}$ & $0.48^{\mathrm{j}}$ & $0.39^{\text {gh }}$ & $71.00^{\text {hij }}$ & $58.00^{\text {ghi }}$ & $47.00^{\text {tghi }}$ & $17.75^{\text {hij }}$ & $14.50^{\text {ghi }}$ & $11.75^{\text {tghi }}$ \\
\hline $\mathrm{kg} 58$ & $3.85^{\mathrm{q}}$ & $3.25^{\mathrm{n}}$ & $2.80^{\mathrm{n}}$ & 0.63 & 0.630 .63 & $0.49^{\mathrm{i}}$ & $0.41^{\mathrm{i}}$ & $0.35^{\mathrm{fg}}$ & $77.00^{\mathrm{ijk}}$ & $65.00^{\mathrm{ijk}}$ & $56.00^{\mathrm{ijk}}$ & $19.25^{\mathrm{ijk}}$ & $16.25^{\mathrm{ij}}$ & $14.00^{\mathrm{ijk}}$ \\
\hline $\mathrm{g} 510$ & $4.15^{\mathrm{r}}$ & $3.75^{\circ}$ & $3.10^{\circ}$ & 0.5 & $\begin{array}{ll}50 & 0.50\end{array}$ & $0.42^{\mathrm{h}}$ & $0.38^{\mathrm{hi}}$ & 0.3 & $83.00^{\mathrm{k}}$ & $75.00^{\mathrm{k}}$ & $62.00^{\mathrm{k}}$ & $20.75^{\mathrm{k}}$ & $18.75^{\mathrm{k}}$ & $15.50^{\mathrm{k}}$ \\
\hline Total & 1.80 & 1.50 & 1.32 & 0.69 & $\begin{array}{lll}0.69 & 0.69\end{array}$ & 0.36 & 0.31 & 0.27 & 55.02 & 45.30 & 39.50 & 13.76 & 11.33 & 9.88 \\
\hline
\end{tabular}

\section{Evaluation indices of the machine}

Mass of liquid extracted by the machine

Figure 4 shows that the maximum mass of liquid extracted of $3.1,3.9$ and $4.2 \mathrm{~kg}$ in $5 \mathrm{~kg}$ sample of cashew nut shell were obtained at C, B, A (all on w.b.) moisture contents respectively and pressing duration of 10 minutes. Meanwhile, at 2 minutes pressing duration and $14.00-16.99 \%$ (w.b.), $17.00-19.99 \%$ (w.b.), 20.00-22.99\% (w.b.) moisture contents, the mass of liquid extracted for $5 \mathrm{~kg}$ sample were $2.5,2.1$ and $1.8 \mathrm{~kg}$ respectively.

The ANOVA results for the interaction between moisture content, pressing duration and mass of liquid extracted in Table 1 shows that there is significant difference, therefore, since there is significant difference in the interaction of the mass of liquid extracted, pressing duration and the moisture content used, pressing duration of 10 minutes and moisture content of $14.00-16.99 \%$ (w.b.) gave the best mass of liquid extracted. 
Performance evaluation...

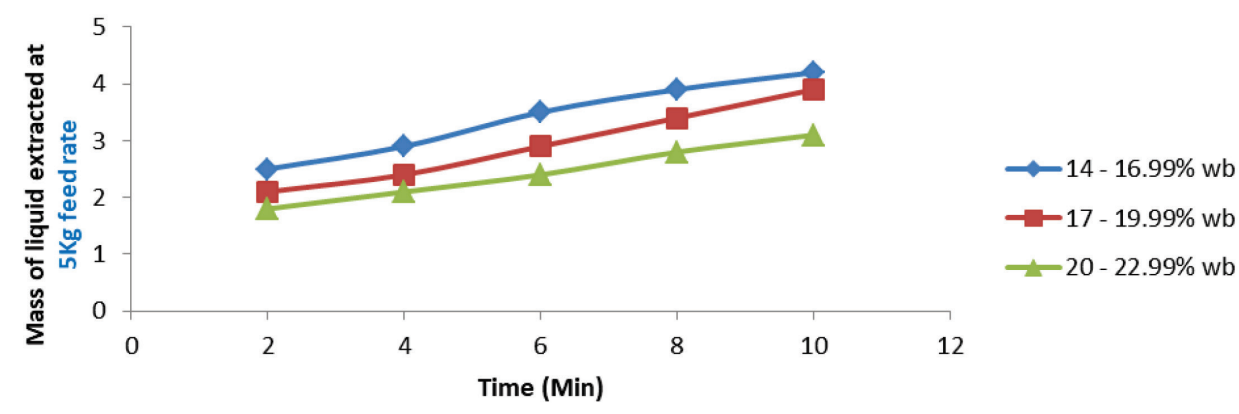

Figure 4. Mass of liquid extracted at $5 \mathrm{~kg}$ feed rate.

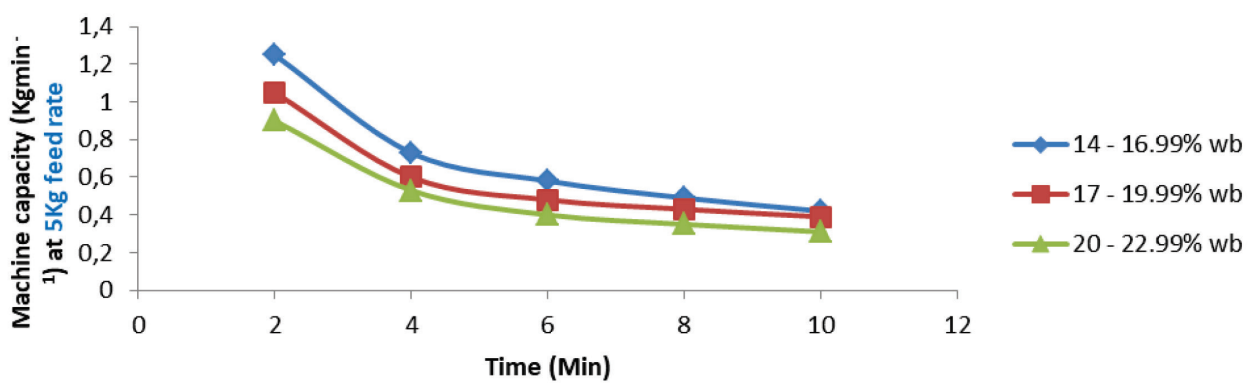

Figure 5. Machine capacity at $5 \mathrm{~kg}$ feed rate.

\section{The capacity of the machine}

Figure 5 shows that the highest machine capacities of $1.25,1.05$ and $0.95 \mathrm{~kg} \cdot \mathrm{min}^{-1}$ were achieved at A, B, C moisture contents and 2 minutes pressing duration respectively for $5 \mathrm{~kg}$ feed rate sample of cashew nut shell while for $5 \mathrm{~kg}$ sample at 10 minutes pressing duration and $\mathrm{A}$. B, C moisture contents, the machine capacities were $0.41,0.36$ and $0.31 \mathrm{~kg} \cdot \mathrm{min}^{-1}$ respectively.

The result from the ANOVA analysis table in Table 1 reveals that there is significant difference in the interaction between machine capacity, moisture content and pressing duration. Pressing duration of 2 minutes and moisture content of group A gave the best machine capacity.

\section{Extraction efficiency of the machine}

Figure 6 shows that the maximum extraction efficiency of $84 \%, 78 \%$ and $62 \%$ at A, B $\mathrm{C}$ moisture contents and 10 minutes pressing duration respectively were obtained for $5 \mathrm{~kg}$ sample of cashew nut shell while at 2 minutes and A, B, C moisture contents, the extraction efficiencies were $50 \%, 42 \%$ and $36 \%$ respectively. 


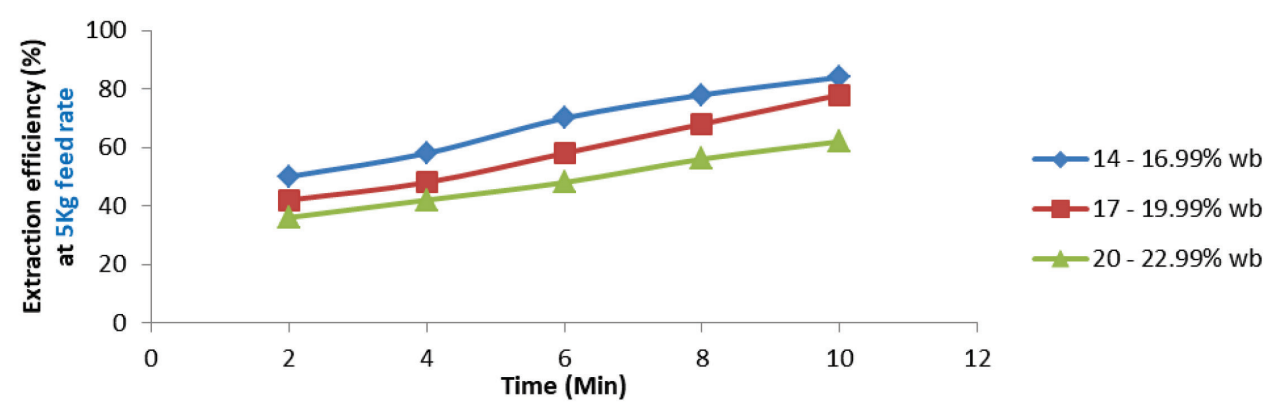

Figure 6. Extraction efficiency at $5 \mathrm{~kg}$ feed rate

The ANOVA results in Table 1 shows that there is significant difference in the interaction between extraction efficiency, moisture content and pressing duration. So the moisture content of group A and pressing duration of 10 minutes gave the best extraction efficiency.

\section{Percntage of liquid recovery of the machine}

Figure 7 shows that the highest percentage oil recovery of $21 \%, 19.5 \%, 15.5 \%$ at 10 minutes and $\mathrm{A}, \mathrm{B}, \mathrm{C}$ moisture contents respectively were obtained for $5 \mathrm{~kg}$ sample of cashew nut shell while at 2 minutes pressing duration and $\mathrm{A}, \mathrm{B}, \mathrm{C}$ moisture contents, the percentage oil recovery were $12.5 \%, 10.5 \%$ and $9 \%$ respectively.

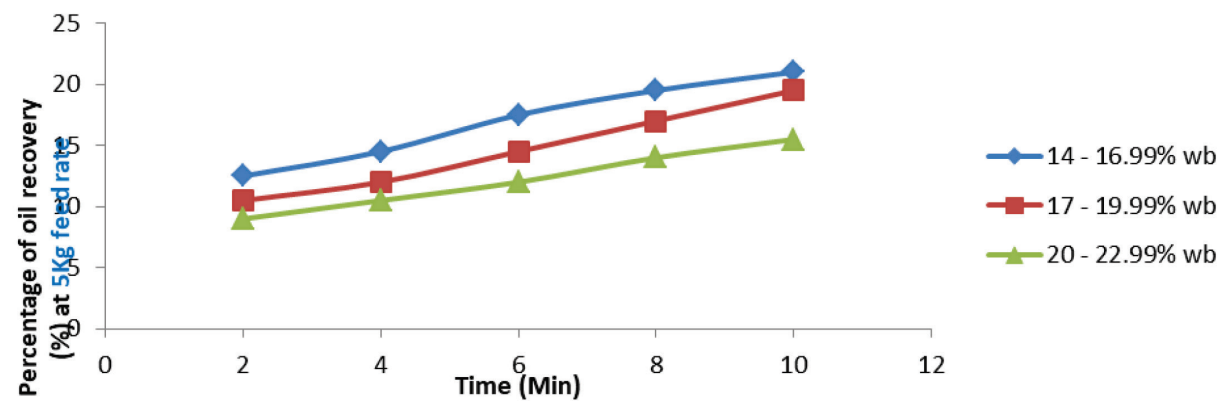

Figure 7. Percentage oil recovery at $5 \mathrm{~kg}$ feed rate

The results of the ANOVA in Table 2 and Duncan multiple range test for machine performance parameters in Table 3 shows that there is significant difference in the interaction between percentage oil recovery, pressing duration and moisture content. Therefore, moisture content of group A and pressing duration of 10 minutes gave the best percentage of oil recovery.

The results obtained from the analysis of variance (ANOVA) of the performance evaluation shows that there is no significant difference in the interaction between feed rate, pressing duration and moisture content. Therefore, since there is no significant difference in the interaction between feed rate, pressing duration and moisture content, the machine can be fed with cashew nut shell without giving consideration to the quantity fed in per time. 
Performance evaluation...

The critical operating parameters of the cashew nut shell liquid expeller were presented in Table 4 for optimum machine settings. The pressing duration of 10 minutes and moisture content of A group gave the best mass of liquid extracted, best extraction efficiency and best percentage of recovery. However, pressing duration of 2 minutes and moisture content A gave the best machine capacity. The optimum mass of liquid extracted was $4.1 \mathrm{~kg}$ at optimum machine of $0.5 \mathrm{~kg} \cdot \mathrm{min}^{-1}$ with the optimum extraction efficiency of $82 \%$. The optimum percentage of liquid recovery was $20.5 \%$ for sample mass $5 \mathrm{~kg}$ and pressing duration of 10 minutes respectively.

Table 4

Critical operating parameters of the cashew nut shell liquid expeller

\begin{tabular}{ll}
\hline Performance parameters & Mean values \\
\hline Mass of liquid extracted (kg) & 4.1 \\
Machine capacity (kg/min) & 0.5 \\
Extraction efficiency (\%) & 82 \\
Percentage liquid recovery (\%) & 20.5 \\
Pressing duration (min) & 10 \\
Moisture content (w.b.) & $\mathrm{A}(14.00-16.99)$ \\
Sample mass (kg) & 5 \\
\hline
\end{tabular}

\section{Conclusion}

A cashew nut shell liquid expeller was designed, fabricated and evaluated for its performance. It was found to be efficient in extracting cashew nut shell liquid. Generally, the moisture content of the cashew nut shell and pressing duration influenced the machine performance indices. The mass of liquid extracted, extraction efficiency and percentage oil recovery increased with the increase in the pressing duration and decreased with increase in the moisture content. Meanwhile,the machine capacity decreased with the increase in the moisture content and pressing duration. Pressing duration of 10 minutes and moisture content of $14.00+16.99 \%$ (w.b.) gave the best mass of liquid extracted, best extraction efficiency and best percentage oil recovery. However, pressing duration of 2 minutes and the moisture content of $14.00+16.99 \%$ (w.b.) gave the best machine capacity. The optimum mass of liquid extracted was $4.1 \mathrm{~kg}$ at optimum machine capacity of $0.5 \mathrm{~kg} \cdot \mathrm{min}^{-1}$ with the optimum extraction efficiency of $82 \%$. The optimum percentage of liquid recovery was $20.5 \%$ for sample mass of $5 \mathrm{~kg}$ and pressing duration of 10 minutes respectively. The machine is cheap, as a unit production cost is N75,000, easy to operate and maintain because the parts can be sourced locally. 
E.O. Aina, A.F. Adisa, T.M.A. Olayanju, S.O. Ismaila

\section{References}

Adisa, A. F. (2011). Performance and Assessment of Farm Equipment Utilization in a Nigeria Arable Farm. Journal of Natural Sciences, Engineering and Technology (JNSET), Abeokuta, Nigeria; $10(2), 158-168$.

Akinhanmi, T. F., Atasie, V. N. and Akintokun, P. O. (2008). Chemical Composition and Physicochemical Properties of Cashew nut (Anacardium occidentale) Oil and Cashew nut Shell Liquid Agricultural Engineering, 32, 75-80.

Akinoso, R. (2006). Effects of Moisture Content, Roasting Duration and Temperature on Oil Yield and Quality of Palm Kernel (Elaesis guineesis) and Sesame (Sesamium indicum) Oils. Ph.D Thesis, Department of Agricultural and Environmental Engineering, University of Ibadan, Ibadan, Nigeria.

Akinwale, S. A. and Esan, E. B. (1989). Advances in cashew breeding in Nigeria. In: Progress in Tree Crop Research, 2nd Edition. Cocoa Research Institute of Nigeria (CRIN), Ibadan, Nigeria, 166-174.

ASAE. (1998). Methods of Determining and Expressing the Fineness of Feed Materials by Sieving, ASAE S319-3. American Society of Agricultural Engineers 2950 Niles Road, St. Joseph MI 49085-9659, Michigan, United States of America.

Brunauer, S., Emmett, P.H. and Teller, E. (1999). Adsorption of gases in multimolecular layers. American Journal of Chemical Society, 60, 309-319.

Mandal, R. C. (2012). Cashew Production and Processing technology. Published by Agrobios, behind Nasrani Cinema, Chopsani Road, Jodhpur - 342 003, India, 142-182.

Mazzetto, S. E., Lemonaco, D. and Mele, G. (2009). Validation of methodology for the chemical characterization of sugar cane bagasse. Quim Nova 32, 732.

Ojomo, A. O., Ologunagba, F. O. and Alagha, S. A. (2012). Evaluation of a locally fabricated oil screw expelling machine. Department of Agricultural Engineering Technology Rufus Giwa Polytechnic. Global Journalof Researches in Engineering, 11, 7-10.

Rodrigues, F. H. A., Feitosa, J. P. A.; Ricardo, N. M. P. S., Franca, F. C.Sanwo, B. O.and Kuti J. O. (1972). Cashew Germplasm collections. The Annual Report of Cocoa Research Institute of Nigeria Science 5894, 100-110.

Sengar, S. H. Mohod, A. G. and Khandetod Y. P. (2012). Performance Evaluation of Kiln for Cashew Nut Shell Carbonization and Liquid Department of Electrical and Other Energy Sources. College of Agricultural Engineering and Technology, DBSKKV, Dapoli.

Togun, A. (1977). A review of the prospect of cashew industry. Cocoa Research Institute of Nigeria, Ibadan, pp. 39.

Topper, C. P., Caligari,P. D. S., Camara,M Diaora, S. , Dijaha, A. Coulibali, F, Asante, A. K.Boamah, A.AyodeleE. A. and Adebola., P. O. (2001). West African Regional Cashew Survey Report (Guinea, Guinea Bissau, Cote d'Ivore, Ghana and Nigeria). Sustainable Tree Crop Programme (STCP) and Biohybrids Agrisystem Limited, UK, pp. 1-110. 
Performance evaluation...

\section{OCENA WYDAJNOŚCI PRASY DO TŁOCZENIA OLEJU Z LUPIN NERKOWCA}

Abstrakt. Gęsty żrący olej zawarty w łupinie nerkowca, zwany CNSL, jest znany z niezliczonych zastosowań $\mathrm{w}$ przemyśle chemicznym. Dokonano oceny skuteczności nowej prasy do tłoczenia CNSL w celu wyznaczenia wpływu zawartosci wilgoci i czasu tłoczenia na parametry tłoczenia. Łupiny pogrupowano w następujący sposób: A: 14,00 - 16,99\%, B: 17,00 - 19,99\% i C: $20,00-$ 22,99\% (w.b) zawartości wilgoci w ciągu 2,4,6, 8 i 10 minut czasu tłoczenia oraz kombinacji 1,2,3,4 i 5 kilo łupin nerkowca. Zastosowano analizę wariancji (ANOVA)do analizy statystycznej. Najlepsza masa wytłoczonego oleju, najlepsza wydajność ekstrakcyjna i najlepszy udział procentowy oleju miał miejsce przy czasie tłoczenia wynoszącym 10 minut i zawartości wilgoci 14,00-16,99\% (w.b) a najlepsza wydajność maszyny wystąpiła przy czasie tłoczenia wynoszącym 2 minuty i zawartości wilgoci w przedziale 14,00-16,99\% (w.b.) Wyniki pokazały, że wpływ czasu tłoczenia i zawartości wilgoci na mase wytłoczonego oleju, wydajność maszyny, wydajność tłoczenia oraz procent odzyskania oleju były znaczące i wynosiły 0.05 . Podczas gdy wplyw czasu tłoczenia oraz zawartość wilgoci nie były znaczące dla prędkości posuwu. Wzrost czasu tłoczenia na zmniejszenie zawartości wilgoci spowodował wzrost masy wytłoczonego oleju, wydajności tłoczenia i procent odzyskania oleju podczas gdy wzrost czasu tłoczenia i zawartości wilgoci spowodował spadek wydajnośći maszyny.

Słowa kluczowe:łupina nerkowca, prasa do tłoczenia oleju, pojemność wyjściowa, wydajność tłoczenia. 